\title{
Role of the continuum in reactions with weakly bound systems: A comparative study between the time evolution of a break-up wave function and its coupled-channel approximation
}

\author{
C. H. Dasso ${ }^{1,2}$ and A. Vitturi ${ }^{1,2}$ \\ ${ }^{1}$ Departamento de Física Atómica, Molecular y Nuclear, Facultad de Física, Universidad de Sevilla, Apartado 1065, E-41080 Sevilla, Spain \\ ${ }^{2}$ Dipartimento di Fisica Galileo Galilei and INFN, Via Marzolo 8, I-35131 Padova, Italy
}

(Received 1 April 2009; published 29 June 2009)

\begin{abstract}
We exploit a model describing the breakup of weakly bound nuclei that can be used as a laboratory for testing different prescriptions that have been advanced in the literature to take into account the nearby presence of continuum states. In the model, we follow the evolution of a single-particle wave function in one dimension, initially bound by a Woods-Saxon type potential and then perturbed by a time- and position-dependent external field. Proper choices of this potential can simulate the effect of the interaction between reaction partners in a nuclear collision. These processes generate inelastic excitation probabilities that - distributed over the bound and continuum states of the system-lead to either a partial or a total fragmentation of the final wave function.
\end{abstract}

DOI: 10.1103/PhysRevC.79.064620

PACS number(s): 24.10.Eq, 21.30.Fe, 21.60.Ev, 25.60.Gc

\section{INTRODUCTION}

For well over a decade, we have witnessed a sustained interest in reactions involving weakly bound nuclear systems, in particular, those with projectiles or targets close to the nucleon evaporation lines. Under such conditions, a sudden ejection of one (or more) of their constituent nucleons may take place. These so-called breakup processes are very important, and they have forced us to reexamine closely the role played by the continuum of asymptotically open states that lie close to the particle-emission threshold.

From the point of view of reaction theory, we are no longer talking about a simple binary process but of a quite complex situation that, if strictly approached, would put to a severe test anyone's expertise in the handling of nuclear reaction formalisms.

It is in the nature of things, however, that people immediately made simplifying assumptions and proceeded to implement detailed calculations for the process; for a review of the continuum-discretized coupled-channel (CDCC) method, see, e.g., Ref. [1]. These studies did not just aim to yield simple estimates of the cross sections but have led to quantitative predictions as well [2]. In retrospect-and with benevolence-one can argue that such early analyses fulfilled the double purpose of identifying ingredients of interest and testing the possibilities of different formalisms to reproduce the orders of magnitude shown by the measured cross sections.

In this type of study, one should not, however, be guided exclusively by the ability of a given prescription to produce good fits to the experimental data. To stress this point, we can recall here some experience gained in the area of subbarrier fusion phenomena [3-5]. Over the years, a variety of proposals have been made in the literature, invariably supported by an excellent reproduction of the experimental data at very low bombarding energies. This was so in spite of the fact that it had been established practically from the beginning of the research activity in this field that the highly forbidden character of the tunneling mechanism left enormous room to comply with this requirement [6]. Equally dangerous has been a tendency to "forget"- just by force of custom-the very idealized nature of certain concepts (distribution of fusion barriers comes to mind) and the quite strictly limited range of validity of many approximations of everyday use.

The lesson drawn from that story is that certain crucial issues have to be discussed and understood on their own merits and adopted or rejected by the pure power of reasoning. When unfamiliar approximations are involved (even if they appear obvious and reasonable), it is imperative to check the extent to which they are suitable to function within the new context. To this end, one must construct alternative solutions of the problem that are far less objectionable and test thoroughly the consequences of their implementation.

One certainly wishes to avoid in the study of breakup phenomena some of the mistakes incurred in the subbarrier fusion field, which took (or, rather, are taking) quite long to eliminate. By the incontestable fact that the number of continuum states is indeed infinite, every single calculation performed so far with a coupled-channel formalism has had to resort to some discretization (better or worse) of the space of scattering states. The computing power available at present has normally limited the positive energy states to be accounted for by at most a double-digit number of channels. Is this enough? What can we expect to believe of the results obtained from a calculation performed in a such a restricted space? The shape of the wave function representative of the process? The predicted $Q$-value distribution? Can we develop some feeling about the size of the energy bins that may be appropriate? And what about the effective truncation of the basis to a maximum energy value?

These are too many questions, and it may be difficult to provide satisfactory general answers for all of them. But one thing is clear. We cannot go forward with the current state of affairs without taking a pause to judge how firm is the ground upon which we stand. Otherwise we could go forever producing and believing irrelevant numbers! (Clearly, by the time this paragraph is being written we already know the conclusions reached in this study. Just to give the reader some incentive to continue further, let us say that one has good reasons to argue that the number of channels used so far by 
different authors has been in general too small to generate reliable breakup wave functions.)

The present contribution is motivated and elaborated in the spirit of the considerations sketched above. The model we shall soon describe is rather simple. Yet, we trust that it incorporates enough of the correct ingredients to allow the extrapolation of its findings to other realistic situations. We rush to state that this is not by any means the first time that a time-dependent approach has been used in the context of nuclear breakup processes [7-10]. But we have deliberately restricted ourselves to examining the results extracted from this scheme in close correspondence with those of its coupled-channel counterpart in a totally equivalent problem. This has provided us with a solid tool for judging the reliability of the latter technique. After all, that is - unavoidably and for the foreseeable futurethe only formulation that will be systematically used for the actual interpretation of experimental data. It is precisely the possibility of investigating any aspect of the problem from the two distinct perspectives (and under controlled circumstances) that lends true value to our exercise.

We hope to be able to keep the following presentation at a somewhat pedagogical level. It would be indeed satisfactory if we managed to give to the experimentalists enough background to follow what their theoretical colleagues are struggling through when they cover these topics in either manuscripts or seminars.

Following this Introduction, we describe, in Sec. II, the basic model that will be at the center of our comparative study. Section III deepens our understanding of the time-dependent approach, where the breakup rate is directly associated with the probability flow out of the potential well where the nucleon orbitals are initially localized. Partial and total breakup situations are discussed, and a number of illustrations are presented to familiarize the reader with what are to become our "reference" results. In fact, there are no specific approximations involved in this part of the study. To implement the coupled-channel approach one has, on the other hand, to introduce a discretized basis for the continuum states; just as the research community has been striving to do in connection with the analysis of real experiments. This is discussed in detail in Sec. IV, where we take time to consider different discretization procedures that mimic those already proposed in the literature. The availability of a reference calculation allows us, in Sec. V, to explore systematically, and through a series of examples, different aspects of the accuracy limitations imposed on the coupledchannel formalism. The results obtained question the chances of constructing meaningful coupled-channel wave functions within the framework of the approximations currently in use. A brief summary and some conclusions close the presentation in Sec. VI.

Some of these results have been previously covered in two conference reports $[11,12]$.

\section{FORMULATION OF THE MODEL}

In this section, we look for a model that can provide an adequate representation of a one-nucleon breakup process. Let us briefly review some of the elements that guide our search.
The problem posed should admit solutions in terms of a coupled-channel formalism that resorts to a discretization of the continuum. This is, after all, the situation whose soundness we set out to test and have listed as the main motivation of the whole exercise. But, clearly, it should nevertheless be possible to generate the evolution of the wave function in an alternative manner, unaffected by a questionable handling of the continuum. To such solutions, one could then assign the label of "exact" and they would become the standards of reference to compare with coupled-channel results.

Just in pondering on the initial and final characteristics that the single-particle breakup wave function should incorporate it becomes clear that we need to formulate a time-dependent problem. Indeed, at the very beginning of the process, we envision a stationary situation where the probability of finding the nucleon must be stable and localized in the vicinity of the breakup nucleus. We take that to be our target and place there the origin of the coordinate system. Later-and as a consequence of the increasing interaction with the incoming projectile - the wave function would start modifying its shape and acquiring excitation energy. Under favorable conditions, the wave function may eventually extend its components into the continuum, whereupon a leakage of probability outside of the target area begins to occur. Finally, as the distance between projectile and target grows again very large and their coupling subsides, the collision process will settle into a third phase. This one should not be judged truly stationary in the sense that the fragment of the wave function that has escaped confinement near the origin continues forever traveling outward. The fraction of probability that it carries away, however, does stabilize. This number is expected to be a number anywhere between 0 (no breakup) and 1 (total breakup).

The model should also be simple, so that its solutions turn out to be fast and easy to construct. This is because we intend to use it as the basic source for the numerous checks required to explore different aspects of the two alternative schemes.

Incorporating all the elements listed above, in what follows we set to describe the evolution of a single-particle wave function $\Psi(x, t)$, initially a bound eigenstate of the onedimensional Woods-Saxon potential

$$
V(x)=\frac{V_{0}}{1+\exp [(|x|-R) / a]},
$$

and perturbed by a space- and time-dependent interaction

$$
V_{\text {coup }}(x, t)=V_{C} \exp \left(-t^{2} / 2 \sigma_{t}^{2}\right) \exp \left[-\left(x-x_{0}\right)^{2} / 2 \sigma_{x}^{2}\right] \text {, }
$$

assumed to be factorized in a time-dependent part $V_{\text {coup }}^{t}(t)=$ $V_{C} \exp \left(-t^{2} / 2 \sigma_{t}^{2}\right)$ and a position-dependent part $V_{\text {coup }}^{x}(x)=$ $\exp \left[-\left(x-x_{0}\right)^{2} / 2 \sigma_{x}^{2}\right]$. As we can see, the coupling is for simplicity assumed to be of Gaussian shape. We note, however, that this is not really essential, and the expression (2) can be easily changed-within the present implementation of the model-to any other functional dependence as long as it stays separable. The potential $V_{\text {coup }}(x, t)$ is meant to simulate the inelastic excitation fields resulting from a collision between two heavy ions. In particular, adjusting the widths $\sigma_{t}, \sigma_{x}$, 
the asymmetry parameter $x_{0}$ and the overall strength $V_{C}$, one is able to mockup realistic situations that arise from different collision times, bombarding energies, interaction ranges, distances of closest approach, impact parameters, etc.

A word about the one-dimensional character of our model Hamiltonian. It may at first appear as too much of a simplification to reduce the three dimensions of the ordinary space to just one. However, the standard way to solve coupledchannel problems in three dimensions is by decomposing the spacial wave functions in angular momentum components. The radial wave functions are defined over a one-dimensional space $r$, much as the one taken into account by the variable $x$ in our model (of course, $0<r<\infty$ while $-\infty<x<$ $\infty)$. The polar angles enter the formalism through angular momentum algebra coefficients that affect both wave functions and form factors but do not add essential complexities to the problem. In short, handling the three-dimensional case amounts to manipulating matrices involving a collection of coupled operations with functions of the radial variable $r$. This is technically similar to the situations that we will exploit in our model space and is not expected to impair any conclusions regarding the validity of the approximation schemes.

Having specified our model, in the following sections we proceed to construct solutions of the common problem in terms of (a) the time-dependent Schrödinger equation and (b) a coupled-channel formalism with discretization of the continuum.

\section{TIME-DEPENDENT APPROACH}

We discuss first the time-dependent approach. In this segment of the paper, we should be able to appreciate, materialized in selected numerical solutions for the breakup wave function $\Psi(x, t)$, the characteristic features anticipated in Sec. II.

In this case, the evolution of the wave function follows, naturally, from the time-dependent Schrödinger equation

$$
i \hbar \frac{\partial \Psi(x, t)}{\partial t}=\left[H_{0}(x)+V_{\text {coup }}(x, t)\right] \Psi(x, t),
$$

where

$$
H_{0}(x)=-\frac{\hbar^{2}}{2 \mu} \frac{\partial^{2}}{\partial x^{2}}+V(x)
$$

can be identified as our "unperturbed" Hamiltonian. The equation has to be solved supplying, as an initial condition at time $t=t_{0}$, the wave function $\Psi\left(x, t=t_{0}\right)$. According to what was previously stated, we take as the initial state for the time-dependent integration precisely one of those bound eigenstates of the unperturbed Hamiltonian $H_{0}(x)$, identified by the index $N$, i.e.,

$$
\Psi\left(x, t=t_{0}\right) \equiv \Phi_{N}(x) \exp \left[-i e_{N}\left(t-t_{0}\right) / \hbar\right] .
$$

Note that we have taken the arbitrary time-dependent phase of the bound-state wave functions to vanish for $t=t_{0}$. Naturally, this time is chosen so that $t_{0} \ll-\sigma_{t}$.

In Fig. 1, we show the profile of our potential $V(x)$, for $V_{0}=$ $-50 \mathrm{MeV}, R=4 \mathrm{fm}$, and $a=0.65 \mathrm{fm}$. The mass corresponds

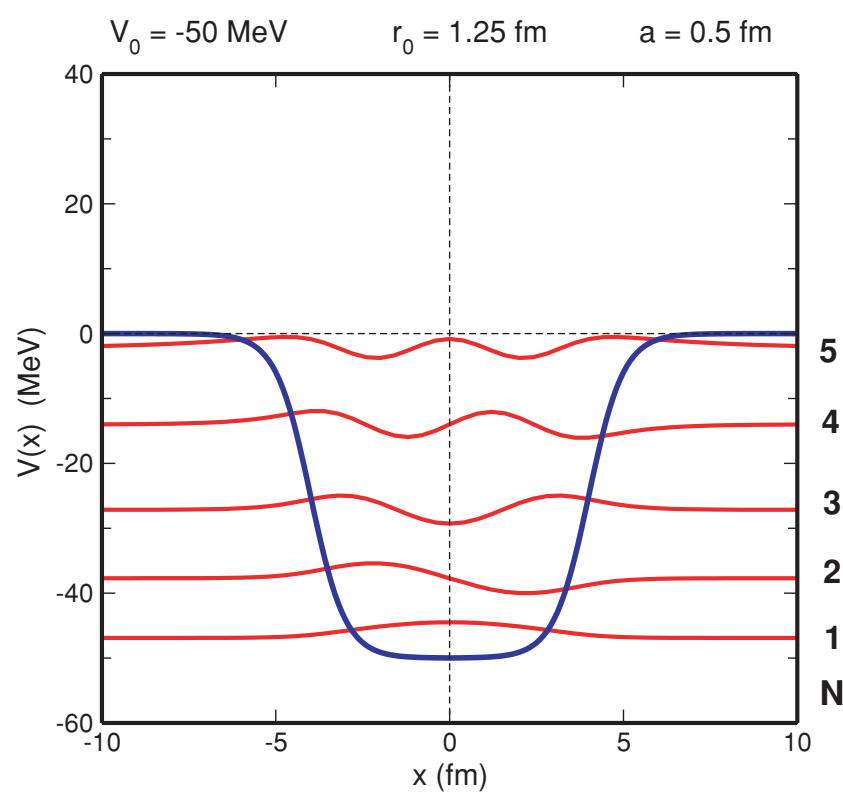

FIG. 1. (Color online) Profile of the Woods-Saxon potential used in the calculations. The parameters chosen are $V_{0}=-50 \mathrm{MeV}, R=$ $4 \mathrm{fm}$, and $a=0.65 \mathrm{fm}$ and are kept fixed for all the illustrations presented in this paper. In the diagram are also shown (in arbitrary scale) the bound-state wave functions $\Phi_{N}(x)$, mounted in the ordinate (in $\mathrm{MeV}$ ) of the corresponding eigenvalue, $e_{N}$. The index $N$ is indicated to the right of the frame.

approximately to that of a neutron, that is, $\mu=1 \mathrm{u}$. The actual values of these constants are of no relevance to the purpose of our comparative study, and we will leave these numbers unchanged for the rest of the presentation. Superimposed onto the figure to constant values equal to the (negative) values of the binding energies $e_{N}$, the bound-state wave functions $\Phi_{N}(x)$ are plotted, in arbitrary units. These are the solutions to the eigenvalue problem

$$
H_{0}(x) \Phi_{N}(x)=e_{N} \Phi_{N}(x)
$$

We can see in the plot that for the parameters chosen, there are just five bound eigenstates. Notice also that the depth of the well, $V_{0}$, was chosen so that the fifth of the neutron states lies very close to the edge of the well. It is, in other words, a "weakly bound state."

As previously stated, the particle is taken initially to be in one of the bound states $\Phi_{N}(x)$ of the Hamiltonian $H_{0}(x)$ shown in Fig. 1. Our first example refers to a case in which one starts with the particle in the lowest energy bound level $(N=1)$ and applies a symmetric perturbation $\left(x_{0}=0\right)$.

The strength of the coupling is here chosen sufficiently weak so that the probabilities of excitation to the upper levels at the end of the collision distribute exclusively within the set of bound states.

Three snapshots of the time evolution of the wave function are shown in Fig. 2. As we can observe, the final wave function is confined to the region of the potential well but is no longer stationary. (Note that we use the term "final" because it is the time $t_{f}$ when we have arbitrarily stopped the calculation. Since - as we said - the wave function is no longer stationary, 


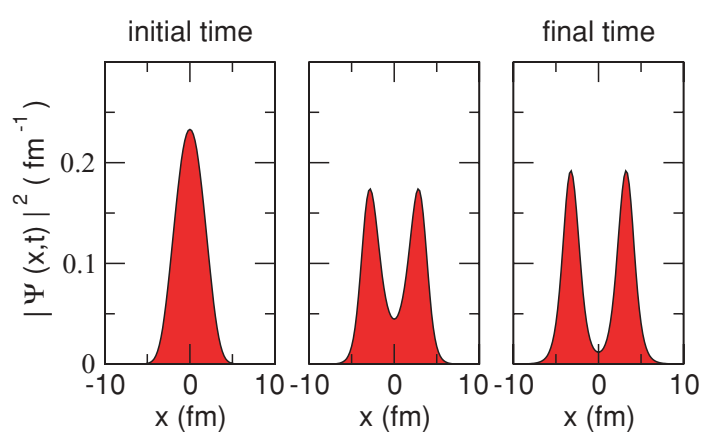

final probability for each bound state

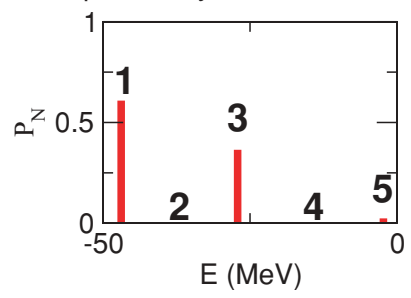

FIG. 2. (Color online) Time evolution of the wave function square (upper part) and final distribution of probability over the discrete bound states (lower part). A symmetric perturbation has been used $\left(x_{0}=0\right)$, starting from the initial $N=1$ state.

it will in fact display a changing pattern forever after the interaction $V_{\text {coup }}$ has ceased to exist; clearly $t_{f}$ must satisfy $\left.t_{f} \gg \sigma_{t}\right)$. The probability distribution over the set of bound states can be obtained by projecting the final wave function, $P_{N}=\left|\left\langle\Phi_{N} \mid \Psi(t \rightarrow+\infty)\right\rangle\right|^{2}$, as shown in the lower part of the figure. Since in this case the perturbation is spatially symmetric, only bound states of the same parity as the initial state can be populated.

When an asymmetric perturbation is used $\left(x_{0} \neq 0\right)$, as in the example shown in Fig. 3, states with both parities can be populated. Yet, also here the external coupling is weak enough so that only the bound states of the eigenvalue spectrum are involved (and, consequently, the sum of the probabilities for these discrete states satisfies $\sum_{N} P_{N} \approx 1$ ).

The situation qualitatively changes if a stronger external field is used. Or, alternatively, if one starts from an initial state closer to the separation threshold. One such example is shown in Fig. 4, where the $N=3$ state is now chosen as the initial configuration. It can be immediately seen that, evolving in time, a major fraction of the wave function "escapes" the potential well by populating unbound states in the continuum. This can be interpreted as describing final breakup events. As the now stronger perturbation was chosen asymmetric $\left(x_{0} \neq 0\right)$, breakup probabilities are different in one direction or the other. We can also see that a fraction of the wave function remains localized in the potential region, corresponding to some finite probability of populating only bound states. The situation can therefore be interpreted as describing a partial breakup process. Going toward the "left" or toward the "right" is the equivalent, in one dimension, to breaking up by emitting the fragment into different orientation angles. The actual situation in the ordinary three-dimensional space is reconstructed - as previously mentioned-from the

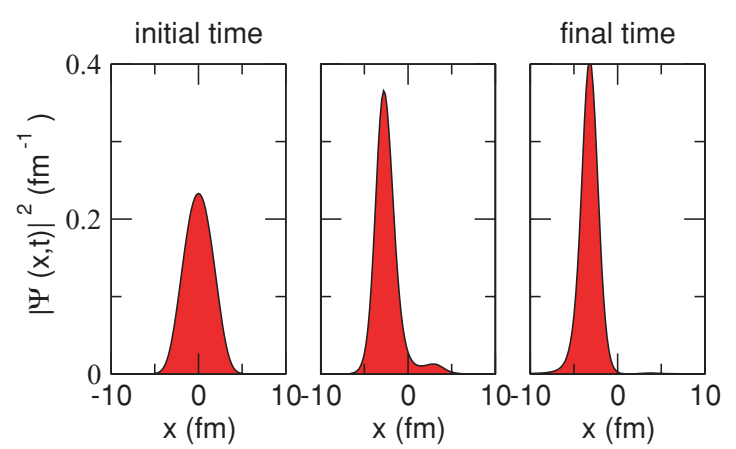

final probability for each bound state

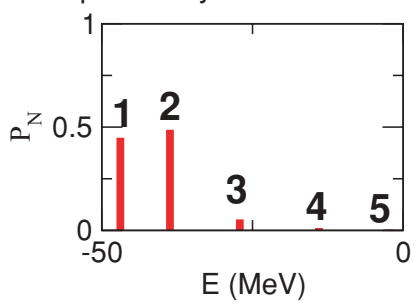

FIG. 3. (Color online) Same as Fig. 2, but for an asymmetric perturbation $\left(x_{0} \neq 0\right)$.

expansion of the incident flux in angular momentum (partial wave) components.

A better insight into the continuum part of the wave function can be obtained by inspecting, besides the modulus square of the wave function, the time evolutions of the probability current and the momentum distribution. One can conclude directly from Fig. 5 that, in fact, the two external wave packets do correspond to fragments moving in opposite directions. One could also show that the two wave packets have momentum distributions with opposite signs. The parts of the wave function associated with the left fragment, the central part, and the right fragment will therefore yield the probability of breakup to the left, no breakup, and breakup to the right, respectively. These quantities are directly related to experimentally measurable quantities.

The situation changes again as one starts the calculation from the last bound orbital $(N=5)$, a condition that one expects to encounter in the vicinity of the drip lines. It is apparent from the evolution of $|\Psi(x, t)|^{2}$ (shown in Fig. 6) that no appreciable part of the wave function remains in this case within the potential region. Notice the expanding scale of the abscissas in the subsequent frames. This is necessary to accommodate the leaking character of the probability out of the finite-size potential range $V_{0}(x)$. There is practically no final overlaps with the bound states, and the situation thus corresponds to a nearly total breakup process. Once again, the characteristics of the process can also be visualized by looking at the current and momentum distributions. These are shown in Fig. 7.

The solution of the problem so far has not involved an expansion of the time-dependent wave function into any basis of single-particle states. In particular, it has not yet been 
initial bound state $\mathrm{N}=3$

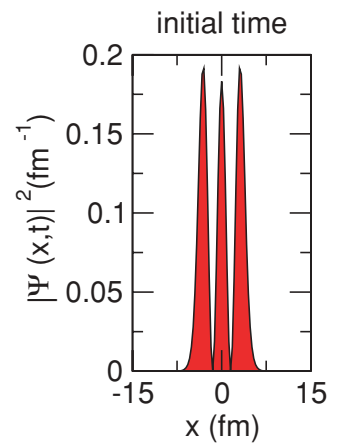

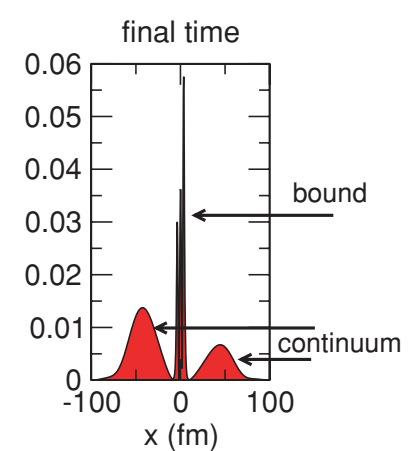

FIG. 4. (Color online) Time evolution of the square of the wave function. An asymmetric perturbation has been used, and the initial configuration corresponds to that of the $N=3$ bound state. Note the increasing range of the abscissas in the different frames; this is necessary to accommodate a distribution of probability that has began to expand outward, both to left and right. As earlier, "final time" means an arbitrary time at which the calculation was stopped. necessary to introduce explicitly any scattering states, since continuum effects are automatically incorporated in $|\Psi(x, t)|^{2}$.

In those cases involving emerging fragments, however, one might like to not only predict the total breakup probability but also learn about the $Q$-value distribution of the emitted nucleons. This amounts to extending to the positive energy axis the probability distributions that in our first two examples (cf. Figs. 2 and 3) were restricted-by the weak character of the coupling - to the bound states only. To this end, one needs to expand the final wave function into a complete set of energy eigenstates of $H_{0}$ including, of course, those lying in the continuum.
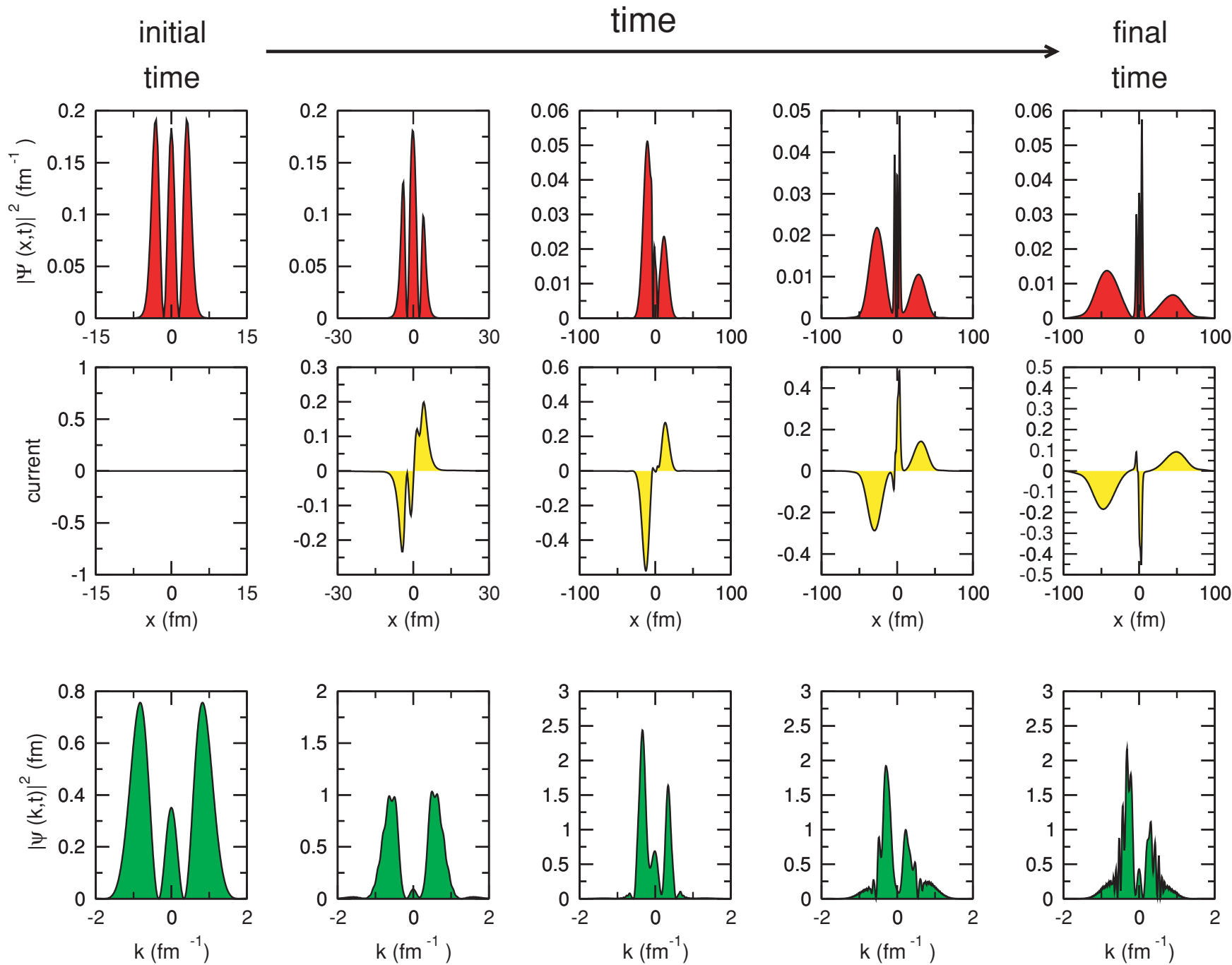

FIG. 5. (Color online) Time evolution of the wave function probability (upper row), current distribution (middle row), and momentum distribution (lower row), associated with the case displayed in Fig. 4. 
initial bound state $\mathrm{N}=5$

initial time

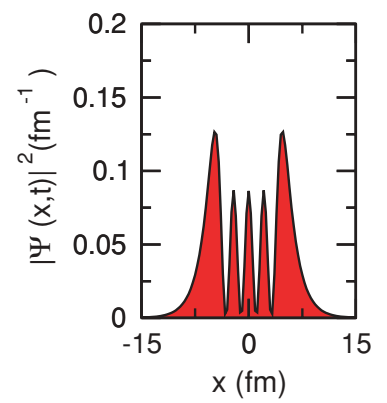

final state: moving frangments

final time
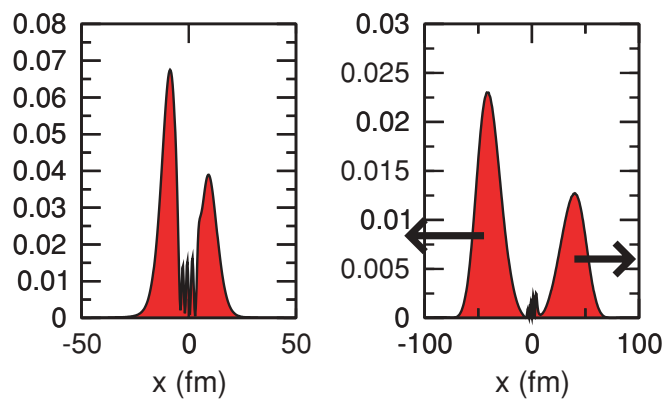

The continuum states used in our calculations need to be orthogonal to the bound states and orthonormal (in a Dirac $\delta$-function sense) between themselves. These conditions by themselves do not define uniquely a basis of eigenstates of $H_{0}(x)$, in particular those with positive energy eigenvalues $E$.
FIG. 6. (Color online) Time evolution of the wave function probability, obtained with an asymmetric perturbation, starting from the initial weakly bound $N=5$ state.
Critical points in this regard are the asymptotic boundary conditions to be imposed. For each positive energy $E$, we have two possible independent solutions whose selection is a priori arbitrary. But the basis of continuum states could also-as an alternative-incorporate any two independent
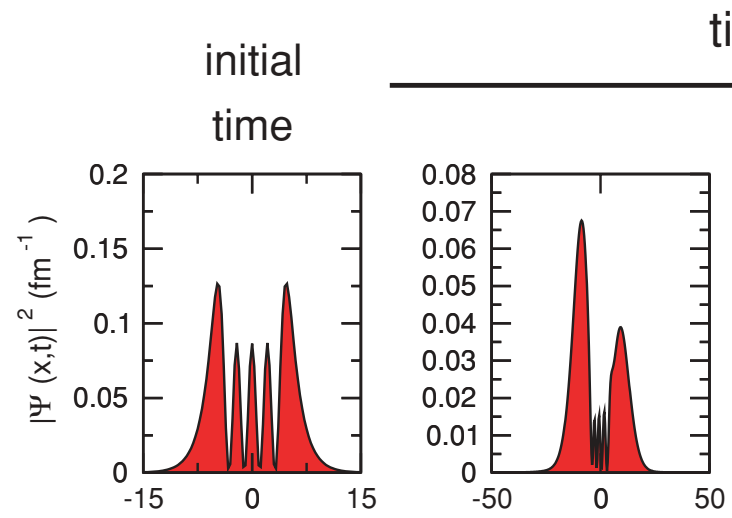

time
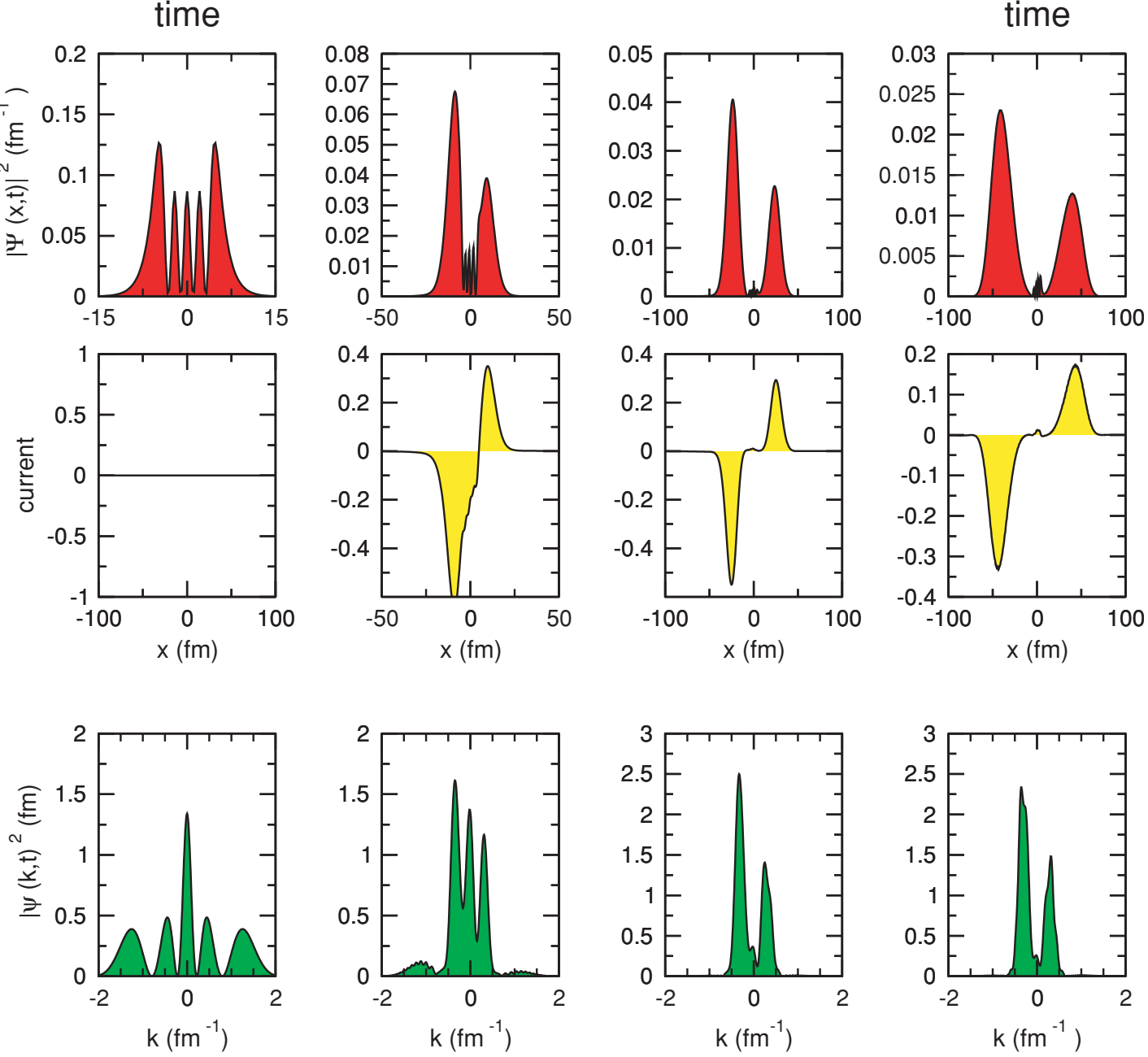

FIG. 7. (Color online) Same as Fig. 5, but for the case displayed in Fig. 6. 


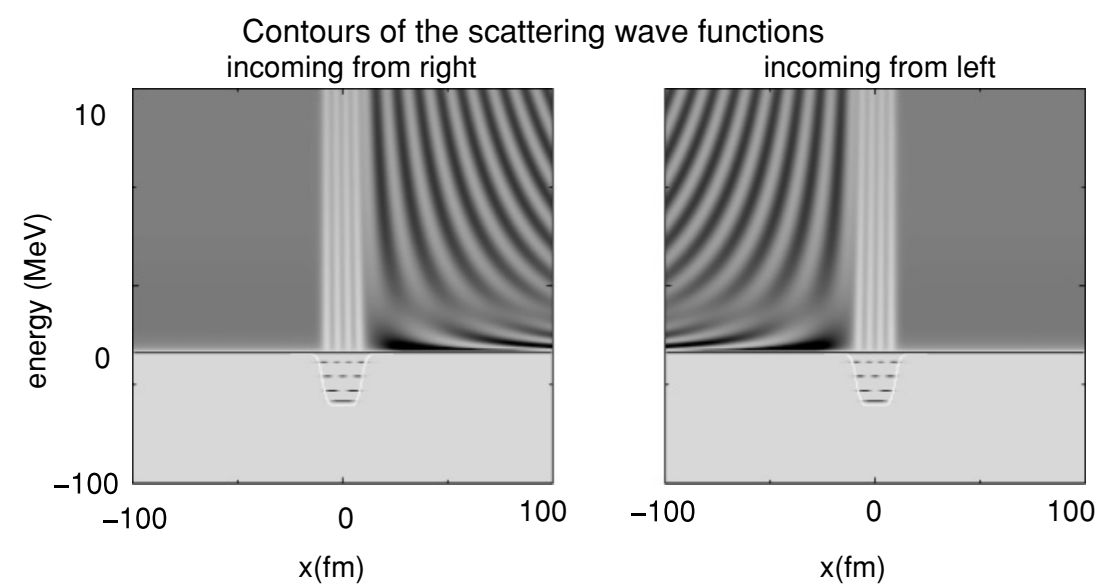

FIG. 8. Contours of the modulus square of the eigenfunctions of $H_{0}(x)$ when they have been chosen and normalized as the scattering states from the right (left frame)and from the left (right frame). Note the different energy scale in the bound and in the continuum sectors, as well as the different normalizations of the bound and continuum wave functions.

linear combinations between the couple of states originally selected.

A possible choice is to take the two solutions corresponding to the scattering process from the right and left sides. Contours corresponding to the modulus square of the scattering wave functions incident from the right are displayed to the left of Fig. 8. Those for incident flux from the left are essentially the same, obtained from Fig. 8 by reflecting the contours by the transformation $x$ into $-x$. Another obvious possible choice could have been to take the symmetric and antisymmetric combinations of these two frames.

The projections and distribution of probabilities of the timedependent solutions of the Schrödinger equation in the basis of eigenstates of the Hamiltonian $H_{0}(x)$ is something that touches closely upon the subjects of our two following sections, and we shall return to the matter-and show many examples-later on.

\section{COUPLED-CHANNEL APPROACH}

As an alternative to the direct numerical solution of the time-dependent Schrödinger equation, one can resort to the familiar coupled-channel formalism, in which the Schrödinger equation is solved by expanding the total wave function on a basis of eigenstates $\Phi_{n}(x, t)$ of the Hamiltonian $H_{0}$. This is the preferred approach in most situations, where the direct numerical solution of the full time-dependent many-body wave function is very complicated. For these reasons it is also the conventional approach used in most of the currently available computer codes. We will use here a simplified version of the coupled-channel scheme, adapted to our one-dimensional problem.

On a discrete basis, the expansion of the full wave function is of the form

$$
\Psi(x, t)=\sum_{n=1}^{\infty} a_{n}(t) \Phi_{n}(x, t),
$$

where the wave functions $\Phi_{n}(x, t)$ are bound eigenstates of the Hamiltonian with the potential $V(x)$ that correspond to the eigenenergy $E_{n}$. The solution of the time-dependent Schrödinger equation reduces in this approach to the solution of the discrete set of coupled equations for the expansion

amplitudes $a_{n}(t)$, that is,

$$
i \hbar \dot{a}_{n}=\sum_{m=1}^{\infty} e^{-i\left(E_{n}-E_{m}\right) t}\left\langle\Phi_{n}\left|V_{\text {coup }}(t)\right| \Phi_{m}\right\rangle a_{m}(t),
$$

which involves the matrix elements of the perturbation potential $V_{\text {coup }}(x, t)$ between the stationary states. The initial conditions for the amplitudes at $t=-\infty$ are fixed by the initial state of the system.

If the basis states are identified with the eigenstates of a finite potential, the complete set of states necessarily includes the discrete bound states and also the continuum states at positive energies. This is indeed the case of the potential (1) considered in this work. In our context-and using the positive energies $E$ as a label for the continuum states, $\Phi(E, x)$-the expansion of the full wave function does in fact incorporate continuum amplitudes $a(E, t)$ in the form

$$
\begin{aligned}
\Psi(x, t)= & \sum_{N=1}^{\infty} a_{n}(t) \Phi_{n}(x)+\int d E\left[a_{-}(E, t) \Phi_{-}(E, x)\right. \\
& \left.+a_{+}(E, t) \Phi_{+}(E, x)\right] .
\end{aligned}
$$

Note: An important word about the units of these continuum wave functions $\Phi_{ \pm}(E, x)$ and reaction amplitudes $a_{ \pm}(E, t)$. Although the notation is deliberately analogous to the one used for their discreet counterparts, one has to be very careful. The nature of the space we are using is such that we are referring to densities in energy. To make things even more confusing, the probabilities are actually obtained from the modulus square of either the $\Phi$ 's or the $a$ 's, so that the units of $\Phi_{+}(E, x)$

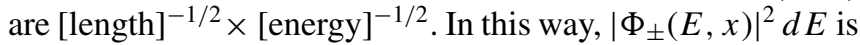
associated with the probability of finding the energy in the interval of energy $[E, E+d E]$. Analogous considerations apply to the amplitudes $a_{ \pm}(E, x)$. According to this discussion, a figure like Fig. 8 has to be studied for a while to realize that the units for the displayed function are actually different for $E>0$ or $E<0$. The same is true for Figs. 9-11.

Besides the standard orthonormality conditions between the discrete bound states, we need to consider also those for the positive and negative continuum states. These wave functions $\Phi(E, x)$ are normalized " $\delta$, , namely,

$$
\left\langle\Phi(E) \mid \Phi\left(E^{\prime}\right)\right\rangle=\delta\left(E-E^{\prime}\right) .
$$


Observe that in the expansion (9) it is explicitly taken into account that for each positive energy $E$, there are two independent solutions. We have chosen these, quite naturally, as the solutions of the scattering problem at energy $E$ with boundary conditions of incoming plane wave from the left $\left(\Phi_{-}\right)$and from the right $\left(\Phi_{+}\right)$. For a symmetric potential, the case of our $V(x)$, the two solutions satisfy the condition $\Phi_{-}(x)=\Phi_{+}(-x)$. But we could have also chosen any other linear combination of these two independent states. For instance, their sum and difference, a choice that yields symmetric and antisymmetric basis states.

With the full (bound plus continuum) basis, the coupled equations for the discrete part of the amplitudes, $a_{n}(t)$ will now include terms that couple not only to the other discrete amplitudes but also to the continuum ones. In addition, we have other coupled equations for the continuum amplitudes $a_{ \pm}(E, t)$, analogous to those of Eq. (8).

All these terms, old and new, are coupled through the matrix elements of the interaction $V_{\text {coup }}(x, t)$. Some of them involve two bound states (bound-bound matrix elements)

$$
V_{n, m}(t)=V_{\text {coup }}^{t}(t)\left\langle\Phi_{n}\left|V_{\text {coup }}^{x}(x)\right| \Phi_{m}\right\rangle ;
$$

others are associated with one bound and one continuum state (bound-continuum matrix elements)

$$
V_{n, \pm}(E)(t)=V_{\text {coup }}^{t}(t)\left\langle\Phi_{n}\left|V_{\text {coup }}^{x}(x)\right| \Phi_{ \pm}(E)\right\rangle ;
$$

and, finally, the remaining couplings involve two continuum states (there are four types,,,+++--+ , and -- of these continuum-continuum matrix elements)

$$
V_{ \pm, \pm}\left(E, E^{\prime}\right)(t)=V_{\text {coup }}^{t}(t)\left\langle\Phi_{ \pm}(E)\left|V_{\text {coup }} X(x)\right| \Phi_{ \pm}\left(E^{\prime}\right)\right\rangle .
$$

The strengths of the coupling form factors [having factorized out the time-dependent part $\left.V_{\text {coup }}^{t}(t)\right]$ are shown as color contour plots in Fig. 9. The phases of the bound states have been chosen so that the corresponding wave functions are real. As a consequence, the bound-bound matrix elements have vanishing imaginary parts. On the other hand, continuum wave functions (and associated matrix elements) are complex, and in
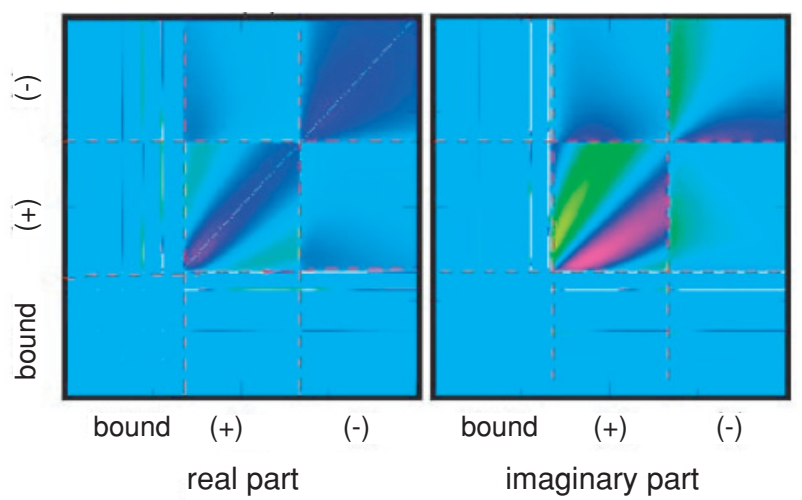

FIG. 9. (Color online) Matrix elements of the coupling interaction $V_{\text {coup }}^{x}(x)$ in the stationary basis. Note the different energy scale in the bound and in the continuum sectors, as well as the different normalizations of the bound and continuum wave functions. Real and imaginary parts of the matrix elements are displayed in the left and right frames.
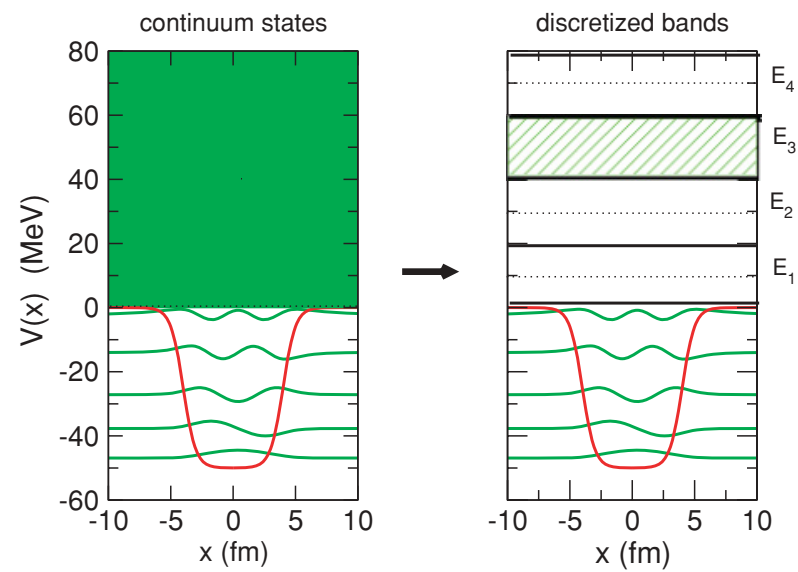

FIG. 10. (Color online) The same five bound eigenstates of the potential $V(x)$ seen in Fig. 1, but on the left, they are shown with a shaded area for the positive energies that represents the infinitely dense set of continuum states. On the right, this same area of positive energy scattering states is replaced by a finite number of states at energies $E_{1}, E_{2}, E_{3}$, etc. These excitation energies are the ones of the representative channels, which are meant to accumulate (according to two different prescriptions, see method 1 , method 2) the excitation strength corresponding to all the states within the bands. One such band is indicated at energy $E_{3}$ with the hatched area.

the figure we separately plot the real and imaginary parts of the couplings. The nine sectors represent different combinations of the bound and the two $( \pm)$ continuum sets. In the bound-bound sector-having only five bound states-there is a total of $5 \times 5=25$ matrix elements (naturally, hardly visible points). In the bound-continuum (and continuum-bound) sectors, the relevant matrix elements lie on horizontal and vertical lines, parallel to the axes. Finally, in the remaining continuumcontinuum sectors, both energies are positive and the matrix elements densely cover the entire sectors.

So far we have not performed any sort of energy discretization. But to solve the continuum differential set of equations, it is imperative to reduce the channels to a finite (preferably small) number. Different prescriptions have been proposed to provide a discrete basis. A first simple procedure (hereafter called method 1) consists in slicing the energy continuum in equally spaced bands, as it is pictorially shown in Fig. 10. Each slice, assumed to be associated with a single state, is then chosen to be represented by the true continuum wave function corresponding to the central energy of the band. The norm of each of those effective discrete "states" must be weighted by a factor $\sqrt{\Delta E}$ to restore the correct units for the distribution of continuum probabilities over the chosen energy mesh of size $\Delta E$.

Note that one could have also started with an alternative expansion to Eq. (9) but in momentum $k$, instead of energy $E$, and thus exploited reaction channels equally spaced in momentum bands. Such a procedure introduces a comparatively larger number of channels in the lower part of the energy spectrum and, for this reason, it is a quite common prescription used in CDCC codes. In either case, the discretized states so defined keep oscillating all the way out to infinity and are not integrable in the usual sense. 
In a second procedure (method 2), again each slice is made to correspond to a single state. But this time the effective configuration is obtained by averaging over the continuum states in the interval $\Delta E$ with some weighting function $w(E)$. Formally,

$$
\Phi_{n}(x)=\frac{1}{\sqrt{\Delta E}} \int_{E_{n}-\Delta E / 2}^{E_{n}+\Delta E / 2} d E w(E) \Phi(E, x)
$$

Advocates of the CCDC program implemented with this particular prescription are always ready to take out a piece of paper and briefly demonstrate how this averaging procedure yields a basis of states normalized to a Kronecker $\delta$ (i.e., $\left\langle\Phi_{n} \mid \Phi_{m}\right\rangle=\delta_{n m}$ ). However, the situation is not entirely satisfactory on at least two accounts.

First, it is motivated by a wish to restrict the spatial range of the wave functions in the basis. (A feature that is not so obvious to impose!) Sometimes this is justified by statements such as "otherwise, how could one treat long-range couplings like the Coulomb ones?" It is never very wise to recast a formulation so that, without changing the physics, the problem at hand simply "disappears." This provides a false sense of security and may lead to the construction of form factors that are no doubt easier to compute but perhaps also to the evaluation of incorrect probability amplitudes. The long range of the Coulomb couplings is certainly a true, real feature of the interaction, and it may be safer to invest some effort-even at the cost of longer computing times and reduced accuracy-to improve the numerical procedures used for such class of slowly converging integrands.

Secondly, the demonstration mentioned above is not actually correct unless the average is extended over the infinite set of states in the interval. This is as impossible to implement as the coupled-channel formalism in the full continuum space. However large the number of states employed to construct the average function for the energy bin, they nevertheless represent a set of zero measure compared to the one associated with the states left out, and thus the Kronecker $\delta$ will not actually emerge from the operation.

Examples of the discretized wave functions obtained by methods 1 and 2 are shown in Fig. 11. These have to be compared with the ones shown earlier in Fig. 8, which were constructed with a practically invisible energy mesh. Different band widths have been used to cover the energy interval up to $10 \mathrm{MeV}$. One can see how the second procedure (method 2) indeed leads to an apparent decay of the wave function for large distances that becomes faster as the relative admixture of wave numbers gets richer. Notice, however, that by construction these functions are, strictly speaking, periodic functions.

With the adoption of a continuum discretization prescription, the form factors involving positive-energy basis states also become discrete. However, approaching the true continuum through more and more precisely defined bands (i.e., decreasing the value of $\Delta E$ ), the overall patterns of the couplings (and their numerical values) should remain stable. This is shown as an example in Fig. 12 for an asymmetric coupling.

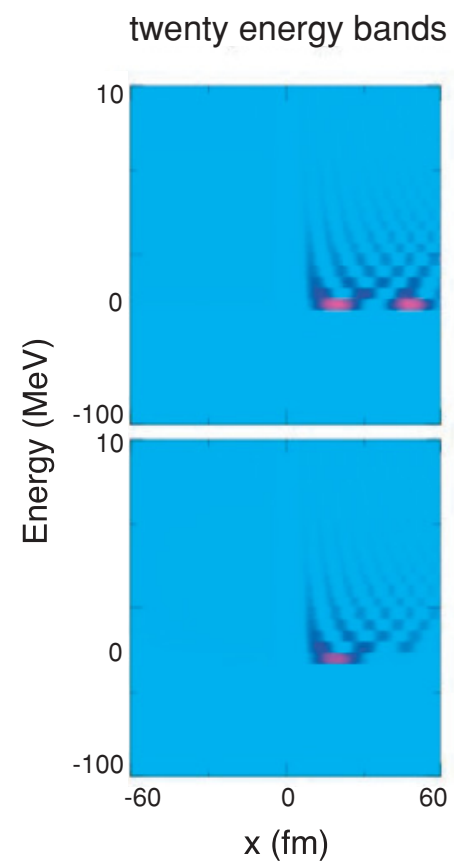

five energy bands
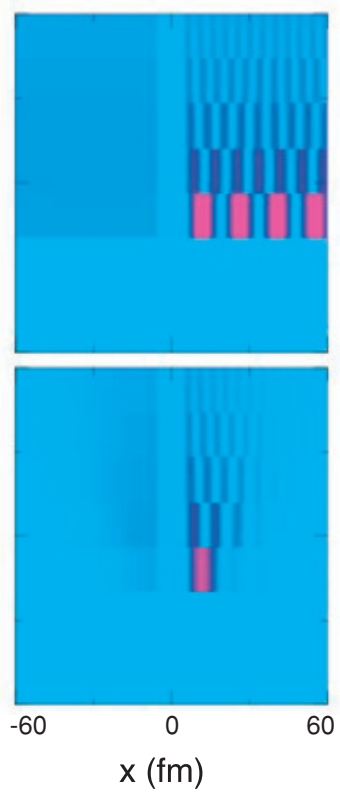

FIG. 11. (Color online) Contours of the modulus square of the bound and continuum discretized wave functions. For the latter, method 1 is used in the two top frames, method 2 in the two lower frames. For both cases we have chosen two different band widths $(0.5$ and $2.0 \mathrm{MeV}$ ). Note the different energy scale in the bound and in the continuum sectors.
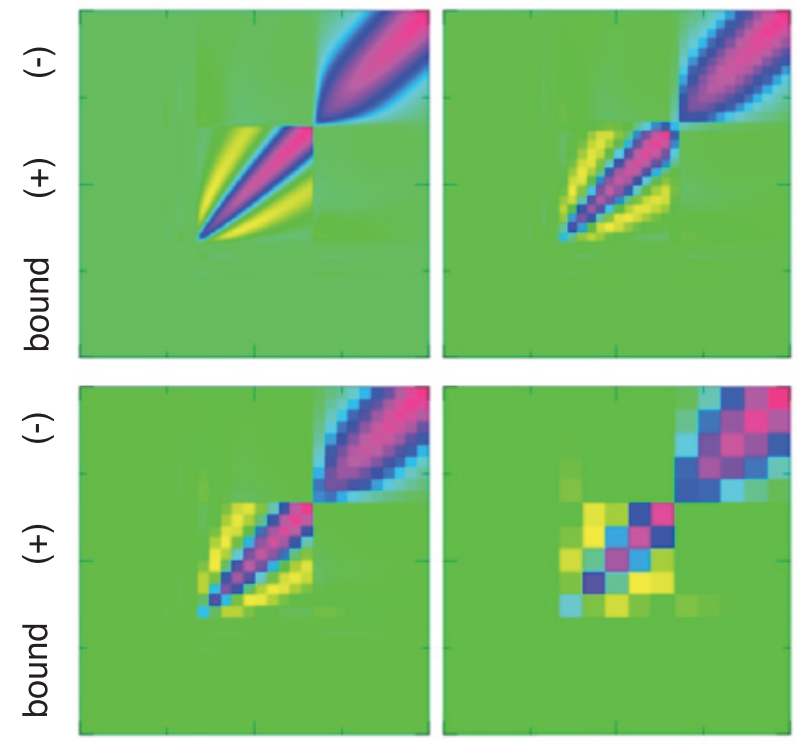

bound

$(+)$

$(-)$

bound

$(+)$

$(-)$

FIG. 12. (Color online) Real part of the coupling form factors of the interaction $V_{\text {coup }}^{x}(x)$ in the stationary basis. Note the different energy scale in the bound and in the continuum sectors. The different frames correspond to different (and increasing) energy meshes in the definition of the discretized continuum states. The top left frame corresponds to the matrix elements in the true continuum basis. 


\section{SYSTEMATIC COMPARISONS}

In this section, we compare the results obtained from the direct evaluation of the breakup wave function, as described in Sec. III, with those obtained in its coupled-channel approximation. The comparison will focus on the $Q$-value distribution of the final breakup wave function. In the former case, this is obtained by projecting the final wave function on the set of basis states (bound plus continuum). For the positive energy part, this gives rise to a continuum $Q$-value distribution, without the need of actually implementing a continuum discretization. In all subsequent figures, these continuum $Q$-value distributions are reported as the solid yellow curves. In the coupled-channel approach, on the other hand, the projection is done over the discretized states we consider and are directly constructed from the final amplitudes $a_{n}$. The corresponding $Q$-value distribution will therefore consist of discrete lines, whose number and position will depend on the parameters chosen for the discretization procedure.

\section{A. Size of the space}

The first relevant parameter is the energy mesh to define the discretized continuum states. The comparison to the reference calculation with the coupled-channel results for the two considered cases is given in Figs. 13 and 14, for different choices of the energy mesh (and consequently different number of basis states). In the comparison, the maximum energy $E_{\text {cut }}$ is kept fixed. We can see from the figures that even in this

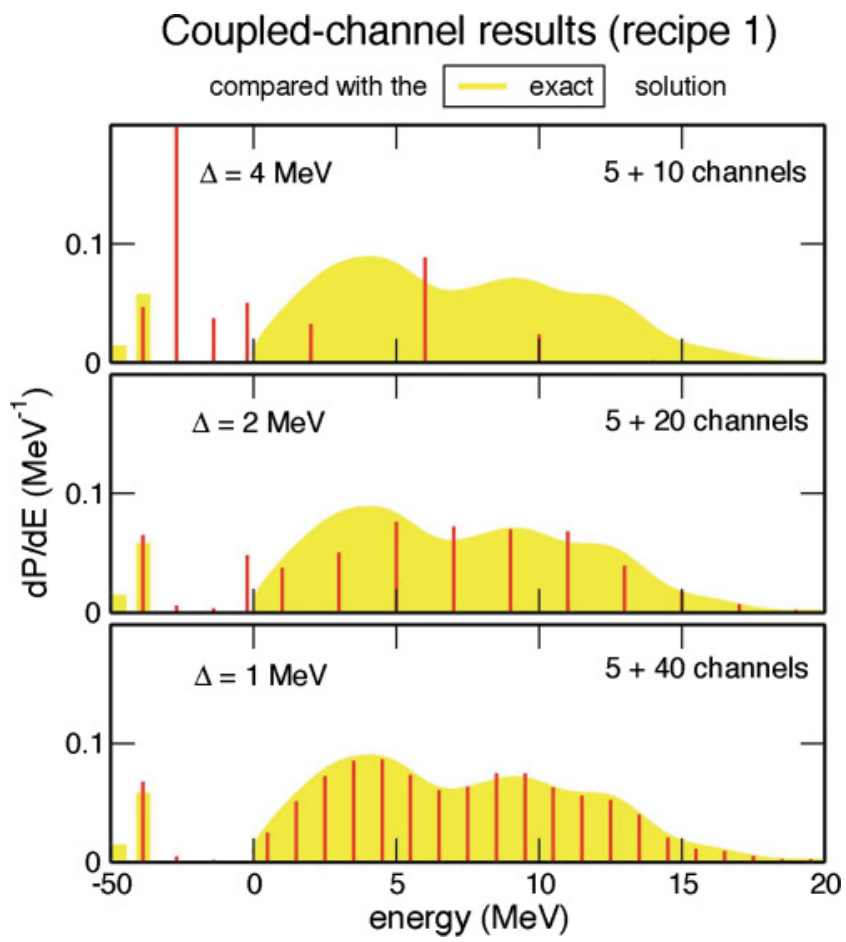

FIG. 13. (Color online) Case of partial breakup starting from a well-bound orbital $(N=3)$. Comparison of final $Q$-value distribution obtained in the exact calculation and with the discretized continuum (method 1). In the latter case, different energy meshes are used in the different frames. The energy cutoff was fixed at $20 \mathrm{MeV}$.

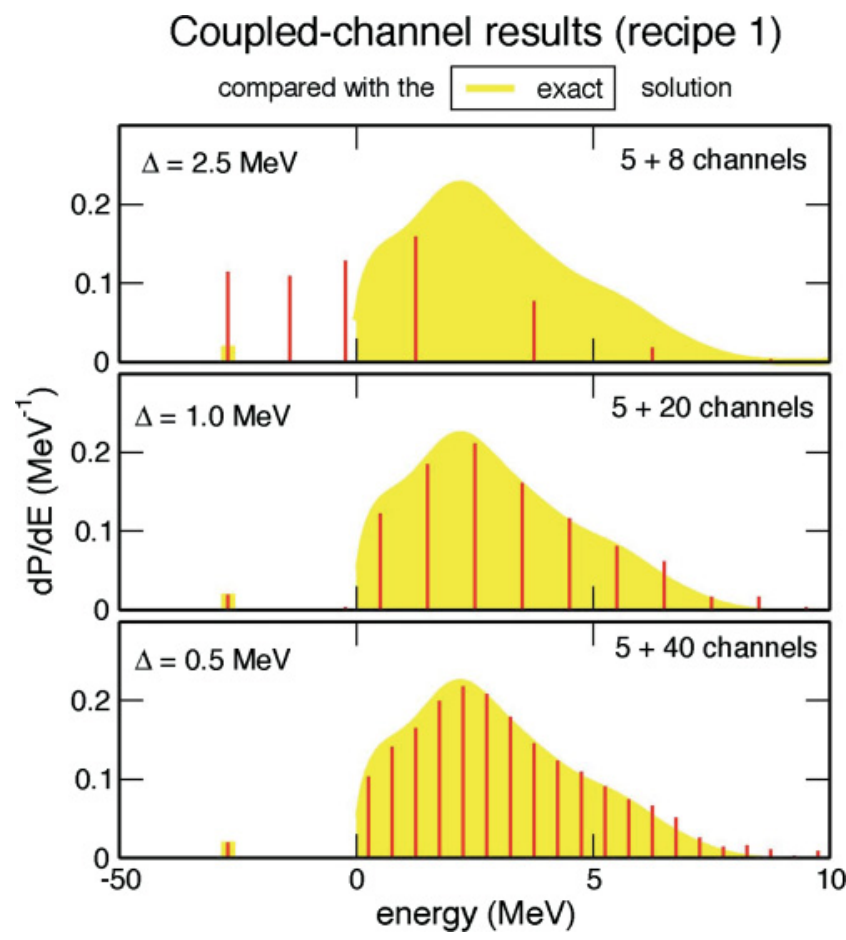

FIG. 14. (Color online) Same as Fig. 13, but starting from the weakly bound orbital $N=5$ and with $E_{\text {cut }}=5 \mathrm{MeV}$.

unidimensional case, dozens of channels are needed to give a proper account of the process.

\section{B. Truncation of the space}

An essential parameter in the calculation-besides the value of the energy mesh $\Delta E$ - is the value of the maximum considered energy $E_{\text {cut }}$. This is a delicate matter insofar as one does not know a priori how high into the continuum the probabilities of excitation will go, depending on the established couplings. Furthermore, just as it is the case in the excitation of a rotational band, there is not always a clear signature that the results are affected by an underestimation of the extent of the calculational space. The obtained results in a multiple-step situation may appear entirely "legal." There is no easy alternative to checking regularly the stability of the probabilities with respect to a further enlargement of the coupled-channel space. Ironically, it is also problematic to exaggerate the range of energies considered, since the inclusion of highly forbidden channels does introduce serious numerical difficulties. An example of the major change in the quality of the results caused by a poor choice of this cutoff energy is shown in Fig. 15.

\section{Discretization method}

The $Q$-value distribution obtained in the coupled-channel calculations with the two methods for the discretization procedure are compared against the exact calculation in Fig. 16. We can see that in the example proposed in the figure, there is no substantial difference in the quality of either 


\section{Coupled-channel results}

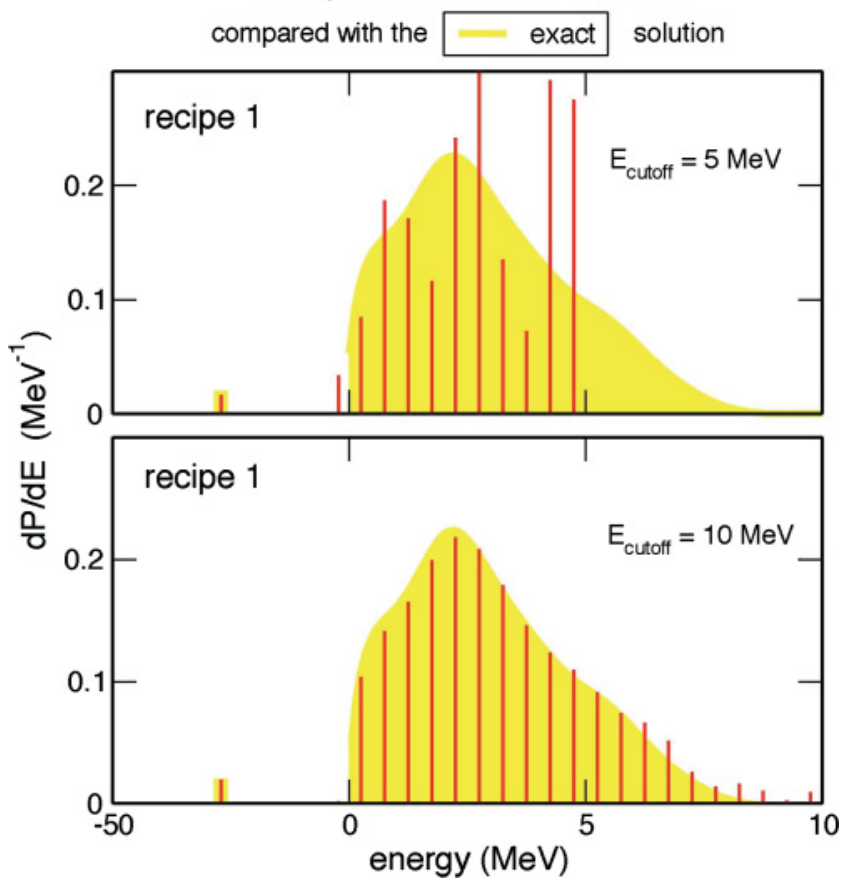

FIG. 15. (Color online) Case of breakup starting from a weakly bound orbital. In the two cases, the exact $Q$-value final distribution is compared with that obtained in the coupled-channel calculation using different energy cutoff for the continuum discretization.

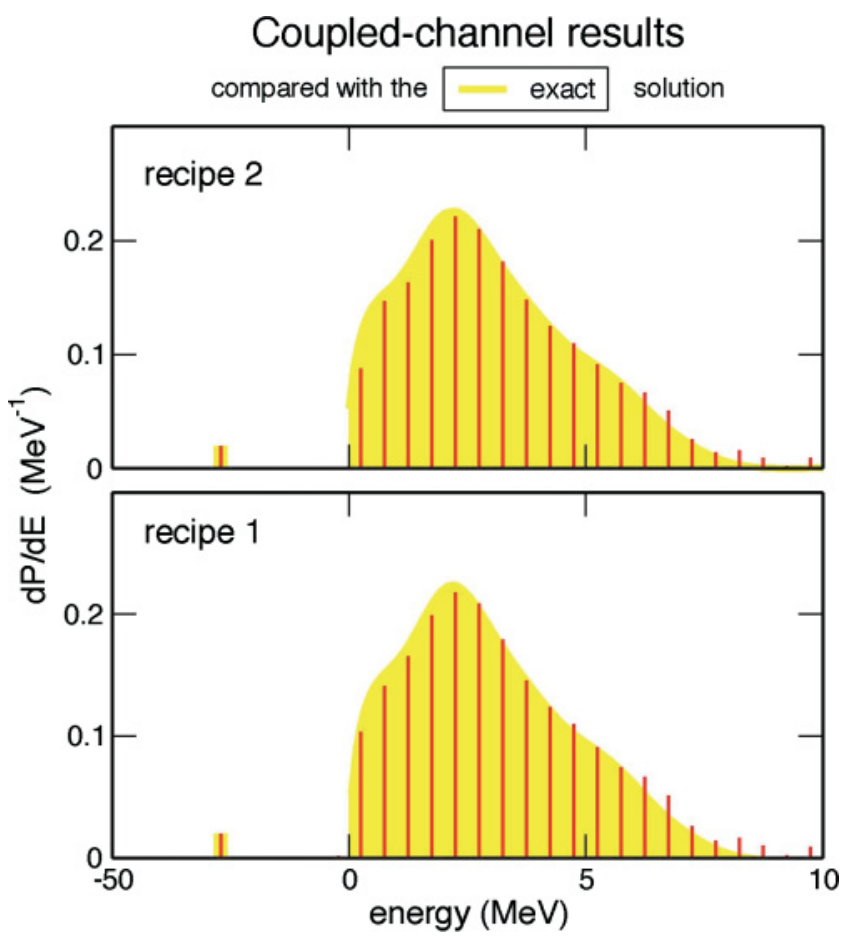

FIG. 16. (Color online) Case of breakup starting from a weakly bound orbital. The exact $Q$-value distribution is compared with those obtained with different prescriptions for the continuum discretization (methods 1 and 2). An energy mesh of $0.5 \mathrm{MeV}$ and an energy cutoff of $10 \mathrm{MeV}$ were used.

\section{Coupled-channel results}

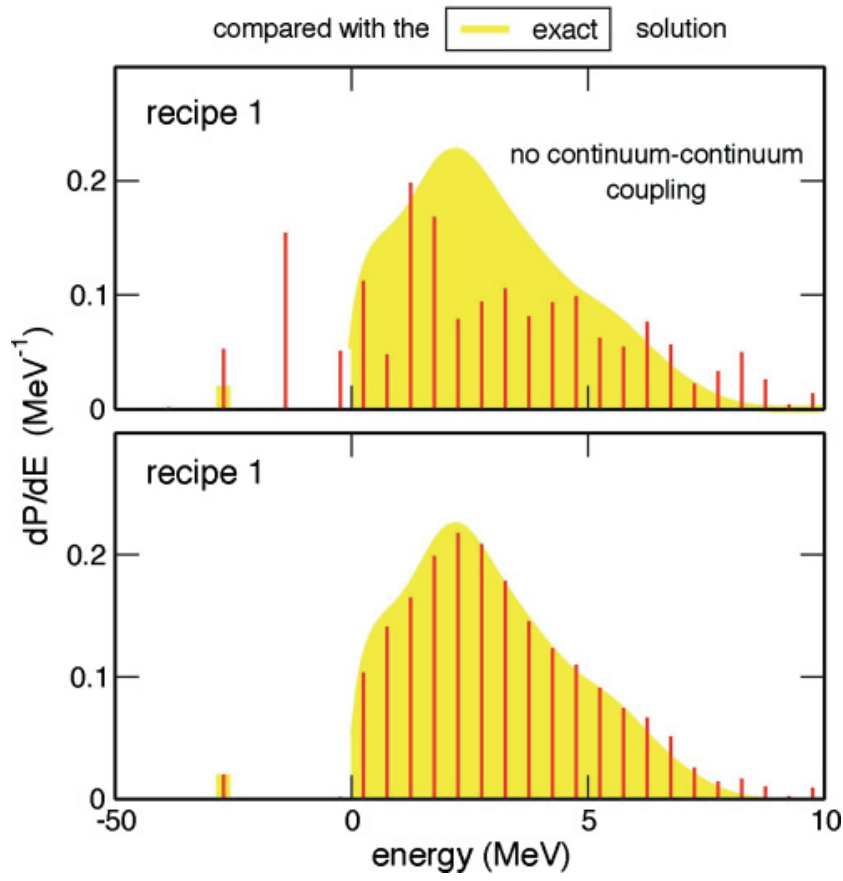

FIG. 17. (Color online) Case of breakup starting from a weakly bound orbital, as in Fig. 14. In the upper frame the continuumcontinuum couplings between continuum states are neglected in the coupled-channel calculation.

approximation. This indicates that there is no major problem handling longer range radial wave functions, as done in method 1 .

\section{Role of multistep and continuum-continuum couplings}

Within the coupled-channel approach, one can easily test different truncations for the coupling matrix elements. There has been speculation on the importance of continuumcontinuum couplings. The importance of these couplings is illustrated in Fig. 17. It is clear from the figure that calculations that exclude the couplings among the discretized continuum states are unable to provide a good description of the process.

In a similar way, one can test the importance of higher order scattering processes by solving just the first-order time-dependent coupled equations. The results, shown in Fig. 18, clearly indicate the inherent multistep character of the excitation mechanism. We would like to emphasize here that some of the conclusions we present in this section have been previously reached in the literature by independent means. For instance, the ones just mentioned in this subsection about the relevance of multistep and continuum-continuum couplings were already discussed in Ref. [13] years ago.

\section{E. Reproduction of the wave function}

Aside from its $Q$-value distribution, it is important to compare directly the evolving exact wave function with its coupled-channel expansion. To this effect we show, in Fig. 19, a comparison between the quality of agreement 


\section{Coupled-channel results}

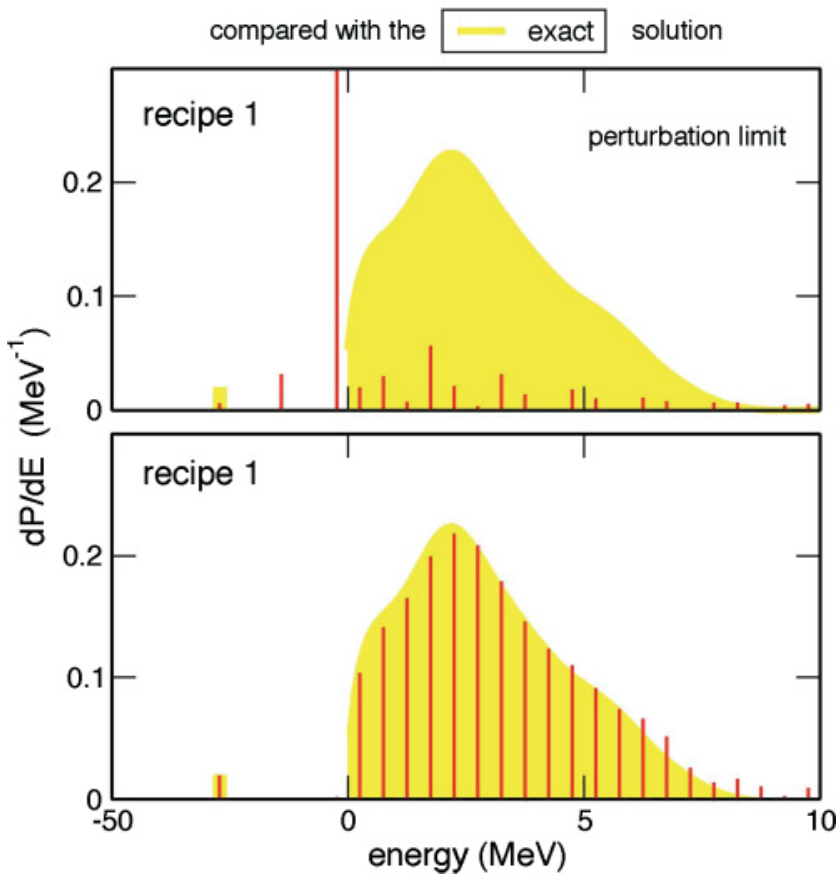

FIG. 18. (Color online) Case of breakup starting from a weakly bound orbital, as in Fig. 14. To study the effect of multistep processes, in the upper frame, we consider the perturbation limit by including in the coupled-channel calculation only the matrix elements between the initial state and all the final states.
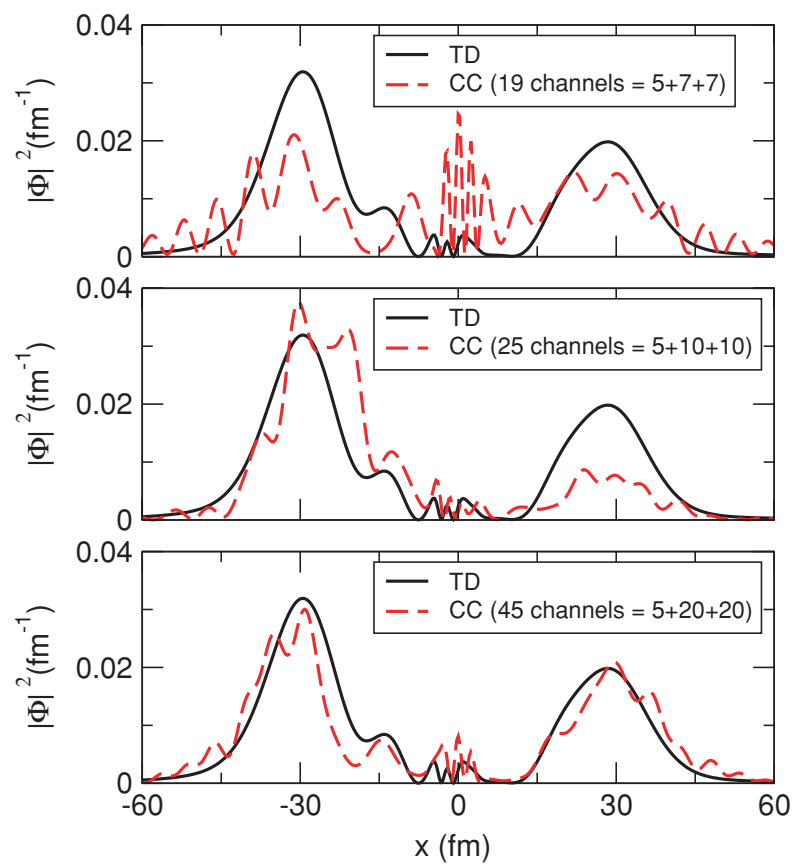

FIG. 19. (Color online) Exact final breakup wave function compared with the one obtained in the discretized coupled-channel calculation. Three cases are shown, corresponding to different choices of the energy mesh in the discretization procedure (and hence different number of effective calculation channels). These correspond to 19 , 25 , and 45 channels from top to bottom. In all cases, the energy cutoff is $10 \mathrm{MeV}$. obtained when the expansion is over 19,25 , or 45 reaction channels. One expects that the precision of the results in the coupled-channel representation will depend on how accurately the derived form factors incorporate all the information that is necessary to follow the evolution of the wave functions as they move over the spatial range of the couplings. For a calculation space with 19 channels, the agreement is quite unacceptable. Only with the larger coupled-channel basis involving 45 channels is the result just beginning to become reasonable. For these plots, we employed method 2 which - as discussed in connection with Fig. 16-is likely to lead to eigenfunctions that display an artificially short spacial range. The consequences that poorly constructed form factors would have in the time evolution of wave functions that do go (in due time) over the incorrectly represented region of $x$ are not so easy to anticipate.

\section{F. Long-range interactions}

Long-range interactions are of interest mostly with regards to choosing the most convenient procedure between methods 1 and 2. A close examination of the agreement for 46 channels shown in Fig. 16 near the threshold shows, if anything, a slightly better result for method 1 . But we should not forget that the range of the interaction used for this illustration was exponentially short. Following the remarks made in the previous section, one could argue that the advantage in favor of method 1 would increase for the case of a long-range interaction like the Coulomb one. This remains here at the level of speculation. But given the fact that most of the early attention in this class of problems was motivated by calculations for $S$ factors of astronomical interest (long-range interactions), learning about the topic may be of more than purely academic interest.

\section{SUMMARY AND CONCLUSION}

In this communication, we have tried to gain a more precise understanding of the limitations that a discretization of the continuum by currently employed methods imposes on the quality of the solutions of a coupled-channel approach to the breakup of weakly bound nuclei. Here we refer to "limitations" in the accuracy of results that are strictly associated with the implementation of the discretization procedure itself and not to the accuracy of the integrating prescriptions that may be exploited to construct the numerical solutions of the problem.

To achieve this task, we have considered it essential to pose a coupled-channel problem that admits the possibility of discretizing the continuum with techniques that are entirely analogous to those used in the literature so far. Let us recall that a breakup process is seen as the inelastic excitation of a nucleon from an initially bound projectile state into any of a set of asymptotically open channels. In this way, a probability flux is established that moves the amplitude of the nucleon motion away from the vicinity of the nucleus and into far away distances.

The microscopic description of such process requires us to follow the evolution in time of a "breakup wave function" 
whose behavior for $t \rightarrow \infty$ specifies different quantities of interest that relate to experimental observables.

At the same time, however, we must have at our disposal an alternative procedure for following the time evolution of the breakup wave function, one that does not rely in any way on a discretization of the continuum. This is what is required to have a reliable standard of comparison to appraise the validity of the coupled-channel approach.

In Sec. II, we introduced a simple model that fulfills the requirements listed above. In Secs. III and IV, we learned how a solution to exactly the same problem can be constructed from either the time-dependent Schrödinger equation or from a coupled-channel formalism.

Our conclusions can be drawn from the series of examples considered in Sec. V, where we compared the results of the discretization of the continuum states exploited in the coupledchannel formalism with the time-dependent formalism, not affected by this approximation.

It is apparent from these illustrations that the reproduction of the breakup wave function is extremely poor if the number of channels is of the orders of magnitudes so far covered in the literature. The distribution of $Q$ values in the continuum is, on the other hand, somewhat better than what could be expected in view of the previous statement. This is somewhat understood for short-range interactions, as it can be argued that the distribution of energies in the continuum has assumed its final form while the colliding nuclei are in close contact.

There is no substantial difference, in our examples, in the quality of the approximations obtained by (method 1$)$ choosing a single representative eigenstate at the middle of the interval or (method 2) averaging many exact wave functions over the intervals of discretization. If anything, method 1 seems to function slightly better. This indicates that there is no major problem handling longer range radial wave functions. The loss of information already at rather short distances artificially created by the averaging method may have, on the other hand, more serious consequences in the case of long-range interactions.

We find the conclusions of this analysis disturbing enough to cast a serious doubt on the indiscriminate use of coupledchannel calculations in the context of nuclear breakup processes. Of course it can be argued that the understanding derived from our model may not be sufficiently strong to abandon altogether the use of this formalism. Specially since - as we already stated earlier-there is no alternative to a coupled-channel formalism to approach this class of continuum problems in the near future.

One could, on the other hand, suspect that the steps necessary to take to make the description more realistic are likely to emphasize the shortcomings already revealed in this preliminary study. Complete CDCC calculations must be posed for each partial wave coupled to a range of transferred angular momenta, with the result of a rapid increase of the dimentionality of the problem and the number of channels involved. Thus, we feel that a more critical approach to the use of these techniques is absolutely imperative and that many more checks along the lines of the ones performed here are necessary to reaffirm our trust in what is being published.

\section{ACKNOWLEDGMENTS}

This work has been partially supported by the Spanish Ministerio de Educación y Ciencia and by the European regional development fund (FEDER) under Project Nos. FIS2008-04189 and FPA2006-13807-C02-01, the Consolider Ingenio Programme CSD2007-0042, the Junta de Andalucia P07-FQM-02894 and FQM160, and the INFN.
[1] J. S. Al-Khalili and F. Nunes, J. Phys. G: Nucl. Part. Phys. 29, R89 (2003).

[2] Not to blame necessarily others we can here quote ourselves, who already published absolute cross sections for the breakup of ${ }^{8}$ B many years ago, C. H. Dasso, S. Lenzi, and A. Vitturi, Nucl. Phys. A639, 635 (1998).

[3] Proceedings of the FUSION97 workshop on heavy-ion collisions at near-barrier energies South Durras, NSW, Australia, J. Phys. G 23, No. 10 (1997).

[4] Proceedings of the International Conference FUSION03: From a Tunneling Nuclear Microscope to Nuclear Processes in Matter Matsushima, Miyagi, Japan, Prog. Theor. Phys. Suppl. 154 (2004).

[5] Proceedings of FUSION06: Reaction Mechanisms and Nuclear Structure at the Coulomb Barrier, Venice, Italy, AIP Conf. Proc. 853 (2006).

[6] R. A. Broglia, C. H. Dasso, S. Landowne, and G. Pollarolo, Phys. Lett. B133, 34 (1983); C. H. Dasso, S. Landowne, and
A. Winther, Nucl. Phys. A405, 381 (1983); A407, 221 (1983).

[7] G. F. Bertsch and C. A. Bertulani, Nucl. Phys. A556, 136 (1993); C. A. Bertulani and G. F. Bertsch, Phys. Rev. C 49, 2839 (1994).

[8] T. Kido, K. Yabana, and Y. Suzuki, Phys. Rev. C 53, 2296 (1996); M. Ito, K. Yabana, T. Nakatsukasa, and M. Ueda, Phys. Lett. B637, 53 (2006).

[9] M. Fallot, J. A. Scarpaci, D. Lacroix, Ph. Chomaz, and J. Margueron, Nucl. Phys. A700, 70 (2002).

[10] P. Capel, G. Goldstein, and D. Baye, Phys. Rev. C 70, 064605 (2004).

[11] C. H. Dasso and A. Vitturi, Nucl. Phys. A787, 476 (2007).

[12] C. H. Dasso and A. Vitturi, in Physics of Unstable Nuclei: Proceedings of the International Symposium ISPUN 07, edited by Dao Tien Khoa, Peter Egelhof, and Sydney Gales (World Scientific, Singapore, 2008), p. 75.

[13] F. M. Nunes and I. J. Thompson, Phys. Rev. C 59, 2652 (1999). 